1 Hacettepe Journal of Mathematics and Statistics

$\bigcap$ Volume 44 (4) (2015), 855-866

\title{
Existence and nonexistence results for a fourth-order discrete Dirichlet boundary value problem
}

\author{
Xia Liu * , Yuanbiao Zhang ${ }^{\dagger}$ and Haiping Shi ${ }^{\ddagger}$
}

\begin{abstract}
In this paper, a fourth-order nonlinear difference equation is considered. By making use of the critical point theory, we establish various sets of sufficient conditions for the existence and nonexistence of solutions for Dirichlet boundary value problem and give some new results. Our results generalize and complement the results in the literature.
\end{abstract}

2000 AMS Classification: 39A10.

Keywords: Existence and nonexistence; Dirichlet boundary value problem; Fourth-order; Mountain Pass Lemma; Discrete variational theory.

Received 12/04/2013 : Accepted 27/03/2014 Doi : 10.15672 /HJMS.2015449433

\section{Introduction}

Difference equations have attracted the interest of many researchers in the past twenty years since they provided a natural description of several discrete models. Such discrete models are often investigated in various fields of science and technology such as computer science, economics, neural networks, ecology, cybernetics, biological systems, optimal control, and population dynamics. These studies cover many of the branches of difference equations, such as stability, attractivity, periodicity, oscillation, and boundary value problems, see $[6,12-14,16,18,19,21,26,27]$ and the references therein.

\footnotetext{
*Oriental Science and Technology College, Hunan Agricultural University, Changsha 410128, China. Corresponding author.

Science College, Hunan Agricultural University, Changsha 410128, China.

Email: xia991002@163.com

${ }^{\dagger}$ Packaging Engineering Institute, Jinan University, Zhuhai 519070, China.

${ }^{\ddagger}$ Modern Business and Management Department, Guangdong Construction Vocational Technology Institute, Guangzhou 510450, China.

This project is supported by the Specialized Research Fund for the Doctoral Program of Higher Eduction of China (No. 20114410110002), National Natural Science Foundation of China (No. 11101098) and Natural Science Foundation of Guangdong Province (No. S2013010014460).
} 
Below $\mathbf{N}, \mathbf{Z}$ and $\mathbf{R}$ denote the sets of all natural numbers, integers and real numbers respectively. $k$ is a positive integer. For any $a, b \in \mathbf{Z}$, define $\mathbf{Z}(a)=\{a, a+1, \cdots\}, \mathbf{Z}(a, b)=$ $\{a, a+1, \cdots, b\}$ when $a<b$. Besides, ${ }^{*}$ denotes the transpose of a vector.

The present paper considers the fourth-order nonlinear difference equation

$$
\Delta^{2}\left(p_{n-1} \Delta^{2} u_{n-2}\right)-\Delta\left(q_{n} \Delta u_{n-1}\right)=f\left(n, u_{n+1}, u_{n}, u_{n-1}\right), n \in \mathbf{Z}(1, k),
$$

with boundary value conditions

$$
u_{-1}=u_{0}=0, u_{k+1}=u_{k+2}=0,
$$

where $\Delta$ is the forward difference operator $\Delta u_{n}=u_{n+1}-u_{n}, \Delta^{2} u_{n}=\Delta\left(\Delta u_{n}\right), p_{n}$ is nonzero and real valued for each $n \in \mathbf{Z}(0, k+1), q_{n}$ is real valued for each $n \in \mathbf{Z}(1, k+1)$, $f \in C\left(\mathbf{R}^{4}, \mathbf{R}\right)$.

In recent years the study of boundary value problems for differential equations develops at relatively rapid rate. By using various methods and techniques, such as fixed point theory, topological degree theory, coincidence degree theory, a series of existence results of nontrivial solutions for differential equations have been obtained in literatures, we refer to $[1-3,5,15,30]$. And critical point theory is also an important tool to deal with problems on differential equations [9,11,20,25,35]. Only since 2003, critical point theory has been employed to establish sufficient conditions on the existence of periodic solutions of difference equations. By using the critical point theory, Guo and Yu [12-14] and Shi et al. [28] have successfully proved the existence of periodic solutions of second-order nonlinear difference equations. We also refer to $[32,33]$ for the discrete boundary value problems. Compared to first-order or second-order difference equations, the study of higher-order equations, and in particular, fourth-order equations, has received considerably less attention (see, for example, [7,8,10,23,24,26,29,31] and the references contained therein). Yan, Liu [31] in 1997 and Thandapani, Arockiasamy [29] in 2001 studied the following fourth-order difference equation of form,

$$
\Delta^{2}\left(p_{n} \Delta^{2} u_{n}\right)+f\left(n, u_{n}\right)=0, n \in \mathbf{Z}
$$

and obtained criteria for the oscillation and nonoscillation of solutions for equation (1.3). In 2005, Cai, Yu and Guo [4] have obtained some criteria for the existence of periodic solutions of the fourth-order difference equation

$$
\Delta^{2}\left(p_{n-2} \Delta^{2} u_{n-2}\right)+f\left(n, u_{n}\right)=0, n \in \mathbf{Z} .
$$

In 1995, Peterson and Ridenhour considered the disconjugacy of equation (1.7) when $p_{n} \equiv 1$ and $f\left(n, u_{n}\right)=q_{n} u_{n}($ see $[23])$.

The boundary value problem (BVP) for determining the existence of solutions of difference equations has been a very active area of research in the last twenty years, and for surveys of recent results, we refer the reader to the monographs by Agarwal et al. $[17,21,27]$. As far as we know results obtained in the literature for the BVP (1.1) with (1.2) are very scarce. Since $f$ in (1.1) depends on $u_{n+1}$ and $u_{n-1}$, the traditional ways of establishing the functional in [12-14,32-34] are inapplicable to our case. As a result, the goal of this paper is to fill the gap in this area.

Motivated by the above results, we use the critical point theory to give some sufficient conditions for the existence and nonexistence of solutions for the BVP (1.1) with (1.2). We shall study the superlinear and sublinear cases. The main idea in this paper is to transfer the existence of the BVP (1.1) with (1.2) into the existence of the critical points of some functional. The proof is based on the notable Mountain Pass Lemma in combination with variational technique. The purpose of this paper is two-folded. On one hand, we shall further demonstrate the powerfulness of critical point theory in the study of solutions for boundary value problems of difference equations. On the other hand, we 
shall complement existing results. The motivation for the present work stems from the recent paper in [7].

Let

$$
\begin{aligned}
& \bar{p}=\max \left\{p_{n}: n \in \mathbf{Z}(0, k+1)\right\}, \underline{p}=\min \left\{p_{n}: n \in \mathbf{Z}(0, k+1)\right\}, \\
& \bar{q}=\max \left\{q_{n}: n \in \mathbf{Z}(1, k+1)\right\}, \underline{q}=\min \left\{q_{n}: n \in \mathbf{Z}(1, k+1)\right\} .
\end{aligned}
$$

Our main results are as follows.

Theorem 1.1. Assume that the following hypotheses are satisfied:

( $p$ ) for any $n \in \boldsymbol{Z}(0, k+1), p_{n}<0$;

(q) for any $n \in \boldsymbol{Z}(1, k+1), q_{n} \leq 0$;

$\left(F_{1}\right)$ there exists a functional $F(n, \cdot) \in C^{1}\left(\boldsymbol{Z} \times \boldsymbol{R}^{2}, \boldsymbol{R}\right)$ with $F(0, \cdot)=0$ such that

$$
\frac{\partial F\left(n-1, v_{2}, v_{3}\right)}{\partial v_{2}}+\frac{\partial F\left(n, v_{1}, v_{2}\right)}{\partial v_{2}}=f\left(n, v_{1}, v_{2}, v_{3}\right), \forall n \in \boldsymbol{Z}(1, k)
$$

$\left(F_{2}\right)$ there exists a constant $M_{0}>0$ such that for all $\left(n, v_{1}, v_{2}\right) \in \boldsymbol{Z}(1, k) \times \boldsymbol{R}^{2}$

$$
\left|\frac{\partial F\left(n, v_{1}, v_{2}\right)}{\partial v_{1}}\right| \leq M_{0},\left|\frac{\partial F\left(n, v_{1}, v_{2}\right)}{\partial v_{2}}\right| \leq M_{0} .
$$

Then the BVP (1.1) with (1.2) possesses at least one solution.

Remark 1.1. Assumption $\left(F_{2}\right)$ implies that there exists a constant $M_{1}>0$ such that $\left(F_{2}^{\prime}\right)\left|F\left(n, v_{1}, v_{2}\right)\right| \leq M_{1}+M_{0}\left(\left|v_{1}\right|+\left|v_{2}\right|\right), \forall\left(n, v_{1}, v_{2}\right) \in \mathbf{Z}(1, k) \times \mathbf{R}^{2}$.

Theorem 1.2. Suppose that $\left(F_{1}\right)$ and the following hypotheses are satisfied:

$\left(p^{\prime}\right)$ for any $n \in \boldsymbol{Z}(0, k+1), p_{n}>0$;

$\left(q^{\prime}\right)$ for any $n \in \boldsymbol{Z}(1, k+1), q_{n} \geq 0$;

$\left(F_{3}\right)$ there exists a functional $F(n, \cdot) \in C^{1}\left(\boldsymbol{Z} \times \boldsymbol{R}^{2}, \boldsymbol{R}\right)$ such that

$$
\lim _{r \rightarrow 0} \frac{F\left(n, v_{1}, v_{2}\right)}{r^{2}}=0, r=\sqrt{v_{1}^{2}+v_{2}^{2}}, \forall n \in Z(1, k) ;
$$

$\left(F_{4}\right)$ there exists a constant $\beta>2$ such that for any $n \in \boldsymbol{Z}(1, k)$,

$$
0<\frac{\partial F\left(n, v_{1}, v_{2}\right)}{\partial v_{1}} v_{1}+\frac{\partial F\left(n, v_{1}, v_{2}\right)}{\partial v_{2}} v_{2}<\beta F\left(n, v_{1}, v_{2}\right), \forall\left(v_{1}, v_{2}\right) \neq 0 .
$$

Then the BVP (1.1) with (1.2) possesses at least two nontrivial solutions.

Remark 1.2. Assumption $\left(F_{4}\right)$ implies that there exist constants $a_{1}>0$ and $a_{2}>0$ such that

$\left(F_{4}^{\prime}\right) F\left(n, v_{1}, v_{2}\right)>a_{1}\left(\sqrt{v_{1}^{2}+v_{2}^{2}}\right)^{\beta}-a_{2}, \forall n \in \mathbf{Z}(1, k)$.

Theorem 1.3. Suppose that $\left(p^{\prime}\right),\left(q^{\prime}\right),\left(F_{1}\right)$ and the following assumption are satisfied:

$\left(F_{5}\right)$ there exist constants $R>0$ and $1<\alpha<2$ such that for $n \in \boldsymbol{Z}(1, k)$ and $\sqrt{v_{1}^{2}+v_{2}^{2}} \geq$ $R$,

$$
0<\frac{\partial F\left(n, v_{1}, v_{2}\right)}{\partial v_{1}} v_{1}+\frac{\partial F\left(n, v_{1}, v_{2}\right)}{\partial v_{2}} v_{2} \leq \alpha F\left(n, v_{1}, v_{2}\right) .
$$

Then the BVP (1.1) with (1.2) possesses at least one solution.

Remark 1.3. Assumption $\left(F_{5}\right)$ implies that for each $n \in \mathbf{Z}(1, k)$ there exist constants 
$a_{3}>0$ and $a_{4}>0$ such that

$\left(F_{5}^{\prime}\right) F\left(n, v_{1}, v_{2}\right) \leq a_{3}\left(\sqrt{v_{1}^{2}+v_{2}^{2}}\right)^{\alpha}+a_{4}, \forall\left(n, v_{1}, v_{2}\right) \in \mathbf{Z}(1, k) \times \mathbf{R}^{2}$.

Theorem 1.4. Suppose that $(p),(q),\left(F_{1}\right)$ and the following assumption are satisfied:

$\left(F_{6}\right) v_{2} f\left(n, v_{1}, v_{2}, v_{3}\right)>0$, for $v_{2} \neq 0, \forall n \in Z(1, k)$.

Then the BVP (1.1) with (1.2) has no nontrivial solutions.

Remark 1.4. In the existing literature, results on the nonexistence of solutions of discrete boundary value problems are scarce. Hence, Theorem 1.4 complements existing ones.

The remainder of this paper is organized as follows. First, in Section 2, we shall establish the variational framework for the BVP (1.1) with (1.2) and transfer the problem of the existence of the BVP (1.1) with (1.2) into that of the existence of critical points of the corresponding functional. Some related fundamental results will also be recalled. Then, in Section 3, we shall complete the proof of the results by using the critical point method. Finally, in Section 4, we shall give three examples to illustrate the main results.

For the basic knowledge of variational methods, the reader is referred to $[20,22,25,35]$.

\section{Variational structure and some lemmas}

In order to apply the critical point theory, we shall establish the corresponding variational framework for the BVP (1.1) with (1.2) and give some lemmas which will be of fundamental importance in proving our main results. Firstly, we state some basic notations.

Let $\mathbf{R}^{k}$ be the real Euclidean space with dimension $k$. Define the inner product on $\mathbf{R}^{k}$ as follows:

$$
\langle u, v\rangle=\sum_{j=1}^{k} u_{j} v_{j}, \forall u, v \in \mathbf{R}^{k},
$$

by which the norm $\|\cdot\|$ can be induced by

$$
\|u\|=\left(\sum_{j=1}^{k} u_{j}^{2}\right)^{\frac{1}{2}}, \forall u \in \mathbf{R}^{k} .
$$

On the other hand, we define the norm $\|\cdot\|_{r}$ on $\mathbf{R}^{k}$ as follows:

$$
\|u\|_{r}=\left(\sum_{j=1}^{k}\left|u_{j}\right|^{r}\right)^{\frac{1}{r}}
$$

for all $u \in \mathbf{R}^{k}$ and $r>1$.

Since $\|u\|_{r}$ and $\|u\|_{2}$ are equivalent, there exist constants $c_{1}, c_{2}$ such that $c_{2} \geq c_{1}>0$, and

$$
c_{1}\|u\|_{2} \leq\|u\|_{r} \leq c_{2}\|u\|_{2}, \forall u \in \mathbf{R}^{k} .
$$

Clearly, $\|u\|=\|u\|_{2}$. For any $u=\left(u_{1}, u_{2}, \cdots, u_{k}\right)^{*} \in \mathbf{R}^{k}$, for the BVP (1.1) with (1.2), consider the functional $J$ defined on $\mathbf{R}^{k}$ as follows:

$$
J(u)=\frac{1}{2} \sum_{n=-1}^{k} p_{n+1}\left(\Delta^{2} u_{n}\right)^{2}+\frac{1}{2} \sum_{n=0}^{k} q_{n+1}\left(\Delta u_{n}\right)^{2}-\sum_{n=1}^{k} F\left(n, u_{n+1}, u_{n}\right),
$$


where

$$
\begin{gathered}
\frac{\partial F\left(n-1, v_{2}, v_{3}\right)}{\partial v_{2}}+\frac{\partial F\left(n, v_{1}, v_{2}\right)}{\partial v_{2}}=f\left(n, v_{1}, v_{2}, v_{3}\right), \\
u_{-1}=u_{0}=0, u_{k+1}=u_{k+2}=0 .
\end{gathered}
$$

Clearly, $J \in C^{1}\left(\mathbf{R}^{k}, \mathbf{R}\right)$ and for any $u=\left\{u_{n}\right\}_{n=1}^{k}=\left(u_{1}, u_{2}, \ldots, u_{k}\right)^{*}$, by using $u_{-1}=$ $u_{0}=0, u_{k+1}=u_{k+2}=0$, we can compute the partial derivative as

$$
\frac{\partial J}{\partial u_{n}}=\Delta^{2}\left(p_{n-1} \Delta^{2} u_{n-2}\right)-\Delta\left(q_{n} \Delta u_{n-1}\right)-f\left(n, u_{n+1}, u_{n}, u_{n-1}\right), \forall n \in \mathbf{Z}(1, k) .
$$

Thus, $u$ is a critical point of $J$ on $\mathbf{R}^{k}$ if and only if

$$
\Delta^{2}\left(p_{n-1} \Delta^{2} u_{n-2}\right)-\Delta\left(q_{n} \Delta u_{n-1}\right)=f\left(n, u_{n+1}, u_{n}, u_{n-1}\right), \forall n \in \mathbf{Z}(1, k) .
$$

We reduce the existence of the BVP (1.1) with (1.2) to the existence of critical points of $J$ on $\mathbf{R}^{k}$. That is, the functional $J$ is just the variational framework of the BVP (1.1) with (1.2).

Let $P$ and $Q$ be the $k \times k$ matrices defined by

$$
\begin{aligned}
P= & \left(\begin{array}{ccccccccc}
6 & -4 & 1 & 0 & 0 & \cdots & 0 & 0 & 0 \\
-4 & 6 & -4 & 1 & 0 & \cdots & 0 & 0 & 0 \\
1 & -4 & 6 & -4 & 1 & \cdots & 0 & 0 & 0 \\
0 & 1 & -4 & 6 & -4 & \cdots & 0 & 0 & 0 \\
\ldots & \cdots & \cdots & \ldots & \cdots & \cdots & \cdots & \cdots & \cdots \\
0 & 0 & 0 & 0 & 0 & \cdots & 6 & -4 & 1 \\
0 & 0 & 0 & 0 & 0 & \cdots & -4 & 6 & -4 \\
0 & 0 & 0 & 0 & 0 & \cdots & 1 & -4 & 6
\end{array}\right), \\
Q & =\left(\begin{array}{ccccccc}
2 & -1 & 0 & \cdots & 0 & 0 \\
-1 & 2 & -1 & \cdots & 0 & 0 \\
0 & -1 & 2 & \cdots & 0 & 0 \\
\cdots & \cdots & \cdots & \cdots & \cdots & \cdots \\
0 & 0 & 0 & \cdots & 2 & -1 \\
0 & 0 & 0 & \cdots & -1 & 2
\end{array}\right) .
\end{aligned}
$$

Clearly, $P$ and $Q$ are positive definite. Let $\lambda_{1}, \lambda_{2}, \cdots, \lambda_{k}$ be the eigenvalues of $P$, $\tilde{\lambda}_{1}, \tilde{\lambda}_{2}, \cdots, \tilde{\lambda}_{k}$ be the eigenvalues of $Q$. Applying matrix theory, we know $\lambda_{j}>0, \tilde{\lambda}_{j}>$ $0, j=1,2, \cdots, k$. Without loss of generality, we may assume that

$$
\begin{aligned}
& 0<\lambda_{1} \leq \lambda_{2} \leq \cdots \leq \lambda_{k}, \\
& 0<\tilde{\lambda}_{1} \leq \tilde{\lambda}_{2} \leq \cdots \leq \tilde{\lambda}_{k} .
\end{aligned}
$$

Let $E$ be a real Banach space, $J \in C^{1}(E, \mathbf{R})$, i.e., $J$ is a continuously Fréchetdifferentiable functional defined on $E . \quad J$ is said to satisfy the Palais-Smale condition (P.S. condition for short) if any sequence $\left\{u^{(l)}\right\} \subset E$ for which $\left\{J\left(u^{(l)}\right)\right\}$ is bounded and $J^{\prime}\left(u^{(l)}\right) \rightarrow 0(l \rightarrow \infty)$ possesses a convergent subsequence in $E$.

Let $B_{\rho}$ denote the open ball in $E$ about 0 of radius $\rho$ and let $\partial B_{\rho}$ denote its boundary.

Lemma 2.1 (Mountain Pass Lemma [25]). Let $E$ be a real Banach space and $J \in$ $C^{1}(E, \boldsymbol{R})$ satisfy the P.S. condition. If $J(0)=0$ and

$\left(J_{1}\right)$ there exist constants $\rho, a>0$ such that $\left.J\right|_{\partial B_{\rho}} \geq a$, and

$\left(J_{2}\right)$ there exists $e \in E \backslash B_{\rho}$ such that $J(e) \leq 0$.

Then $J$ possesses a critical value $c \geq a$ given by

$$
c=\inf _{g \in \Gamma} \max _{s \in[0,1]} J(g(s)),
$$


where

$$
\Gamma=\{g \in C([0,1], E) \mid g(0)=0, g(1)=e\} .
$$

Lemma 2.2. Suppose that $\left(p^{\prime}\right),\left(q^{\prime}\right),\left(F_{1}\right),\left(F_{3}\right)$ and $\left(F_{4}\right)$ are satisfied. Then the functional $J$ satisfies the P.S. condition.

Proof. Let $u^{(l)} \in \mathbf{R}^{k}, l \in \mathbf{Z}(1)$ be such that $\left\{J\left(u^{(l)}\right)\right\}$ is bounded. Then there exists a positive constant $M_{2}$ such that

$$
-M_{2} \leq J\left(u^{(l)}\right) \leq M_{2}, \forall l \in \mathbf{N} .
$$

By $\left(F_{4}^{\prime}\right)$, we have

$$
\begin{aligned}
-M_{2} \leq & J\left(u^{(l)}\right)=\frac{1}{2} \sum_{n=-1}^{k} p_{n+1}\left(\Delta^{2} u_{n}^{(l)}\right)^{2}+\frac{1}{2} \sum_{n=0}^{k} q_{n+1}\left(\Delta u_{n}^{(l)}\right)^{2}-\sum_{n=1}^{k} F\left(n, u_{n+1}^{(l)}, u_{n}^{(l)}\right) \\
\leq & \frac{1}{2} \bar{p} \sum_{n=-1}^{k}\left(u_{n+2}^{(l)}-2 u_{n+1}^{(l)}+u_{n}^{(l)}\right)^{2}+\frac{1}{2} \bar{q} \sum_{n=0}^{k}\left(u_{n+1}^{(l)}-u_{n}^{(l)}\right)^{2} \\
& -a_{1} \sum_{n=1}^{k}\left[\sqrt{\left(u_{n+1}^{(l)}\right)^{2}+\left(u_{n}^{(l)}\right)^{2}}\right]^{\beta}+a_{2} k \\
\leq & \frac{1}{2} \bar{p}\left(u^{(l)}\right)^{*} P u^{(l)}+\frac{1}{2} \bar{q}\left(u^{(l)}\right)^{*} Q u^{(l)}-a_{1} c_{1}^{\beta}\left\|u^{(l)}\right\|^{\beta}+a_{2} k \\
\leq & \frac{1}{2} \bar{p} \lambda_{k}\left\|u^{(l)}\right\|^{2}+\frac{1}{2} \bar{q} \tilde{\lambda}_{k}\left\|u^{(l)}\right\|^{2}-a_{1} c_{1}^{\beta}\left\|u^{(l)}\right\|^{\beta}+a_{2} k,
\end{aligned}
$$

where $u^{(l)}=\left(u_{1}^{(l)}, u_{2}^{(l)}, \cdots, u_{k}^{(l)}\right)^{*}, u^{(l)} \in \mathbf{R}^{k}$. That is,

$$
a_{1} c_{1}^{\beta}\left\|u^{(l)}\right\|^{\beta}-\frac{1}{2}\left(\bar{p} \lambda_{k}+\bar{q} \tilde{\lambda}_{k}\right)\left\|u^{(l)}\right\|^{2} \leq M_{2}+a_{2} k .
$$

Since $\beta>2$, there exists a constant $M_{3}>0$ such that

$$
\left\|u^{(l)}\right\| \leq M_{3}, \forall l \in \mathbf{N}
$$

Therefore, $\left\{u^{(l)}\right\}$ is bounded on $\mathbf{R}^{k}$. As a consequence, $\left\{u^{(l)}\right\}$ possesses a convergence subsequence in $\mathbf{R}^{k}$. Thus the P.S. condition is verified.

\section{Proof of the main results}

In this Section, we shall prove our main results by using the critical point theory.

3.1. Proof of Theorem 1.1

Proof. By $\left(F_{2}^{\prime}\right)$, for any $u=\left(u_{1}, u_{2}, \cdots, u_{k}\right)^{*} \in \mathbf{R}^{k}$, we have

$$
\begin{aligned}
J(u) & =\frac{1}{2} \sum_{n=-1}^{k} p_{n+1}\left(\Delta^{2} u_{n}\right)^{2}+\frac{1}{2} \sum_{n=0}^{k} q_{n+1}\left(\Delta u_{n}\right)^{2}-\sum_{n=1}^{k} F\left(n, u_{n+1}, u_{n}\right) \\
& \leq \frac{1}{2} \bar{p} \sum_{n=-1}^{k}\left(u_{n+2}-2 u_{n+1}+u_{n}\right)^{2}+\frac{1}{2} \bar{q} \sum_{n=0}^{k}\left(u_{n+1}-u_{n}\right)^{2}+M_{0} \sum_{n=1}^{k}\left(\left|u_{n+1}\right|+\left|u_{n}\right|\right)+M_{1} k \\
& \leq \frac{1}{2} \bar{p} u^{*} P u+\frac{1}{2} \bar{q} u^{*} Q u+2 M_{0} \sum_{n=1}^{k}\left|u_{n}\right|+M_{1} k
\end{aligned}
$$




$$
\begin{aligned}
& \leq \frac{1}{2} \bar{p} \lambda_{1}\|u\|^{2}+\frac{1}{2} \bar{q} \tilde{\lambda}_{1}\|u\|^{2}+2 M_{0} \sqrt{k}\|u\|+M_{1} k \\
& \quad \rightarrow-\infty \text { as }\|u\| \rightarrow+\infty .
\end{aligned}
$$

The above inequality means that $-J(u)$ is coercive. By the continuity of $J(u), J$ attains its maximum at some point, and we denote it $\breve{u}$, that is,

$$
J(\check{u})=\max \left\{J(u) \mid u \in \mathbf{R}^{k}\right\} .
$$

Clearly, $\breve{u}$ is a critical point of the functional $J$. This completes the proof of Theorem 1.1 .

\subsection{Proof of Theorem 1.2}

Proof. By $\left(F_{3}\right)$, for any $\epsilon=\frac{1}{8}\left(\underline{p} \lambda_{1}+\underline{q} \tilde{\lambda}_{1}\right)\left(\lambda_{1}\right.$ and $\tilde{\lambda}_{1}$ can be referred to (2.6) and (2.7)), there exists $\rho>0$, such that

$$
\left|F\left(n, v_{1}, v_{2}\right)\right| \leq \frac{1}{8}\left(\underline{p} \lambda_{1}+\underline{q} \tilde{\lambda}_{1}\right)\left(v_{1}^{2}+v_{2}^{2}\right), \forall n \in \mathbf{Z}(1, k),
$$

for $\sqrt{v_{1}^{2}+v_{2}^{2}} \leq \sqrt{2} \rho$.

For any $u=\left(u_{1}, u_{2}, \cdots, u_{k}\right)^{*} \in \mathbf{R}^{k}$ and $\|u\| \leq \rho$, we have $\left|u_{n}\right| \leq \rho, n \in \mathbf{Z}(1, k)$.

For any $n \in \mathbf{Z}(1, k)$,

$$
\begin{aligned}
J(u) & =\frac{1}{2} \sum_{n=-1}^{k} p_{n+1}\left(\Delta^{2} u_{n}\right)^{2}+\frac{1}{2} \sum_{n=0}^{k} q_{n+1}\left(\Delta u_{n}\right)^{2}-\sum_{n=1}^{k} F\left(n, u_{n+1}, u_{n}\right) \\
& \geq \frac{1}{2} \underline{p} \sum_{n=-1}^{k}\left(u_{n+2}-2 u_{n+1}+u_{n}\right)^{2}+\frac{1}{2} \underline{q} \sum_{n=0}^{k}\left(u_{n+1}-u_{n}\right)^{2}-\frac{1}{8}\left(\underline{p}_{1}+\underline{q}_{1}\right) \sum_{n=1}^{k}\left(u_{n+1}^{2}+u_{n}^{2}\right) \\
& \geq \frac{1}{2} \underline{p} u^{*} P u+\frac{1}{2} \underline{q} u^{*} Q u-\frac{1}{4}\left(\underline{p} \lambda_{1}+\underline{q} \underline{\lambda}_{1}\right)\|u\|^{2} \\
& \geq \frac{1}{2} \underline{p} \lambda_{1}\|u\|^{2}+\frac{1}{2} \underline{q} \tilde{\lambda}_{1}\|u\|^{2}-\frac{1}{4}\left(\underline{p} \lambda_{1}+\underline{q}_{1}\right)\|u\|^{2} \\
& =\frac{1}{4}\left(\underline{p} \lambda_{1}+\underline{q}_{1}\right)\|u\|^{2},
\end{aligned}
$$

where $u=\left(u_{1}, u_{2}, \cdots, u_{k}\right)^{*}, u \in \mathbf{R}^{k}$.

Take $a=\frac{1}{4}\left(\underline{p} \lambda_{1}+\underline{q} \tilde{\lambda}_{1}\right) \rho^{2}>0$. Therefore,

$$
J(u) \geq a>0, \forall u \in \partial B_{\rho} .
$$

At the same time, we have also proved that there exist constants $a>0$ and $\rho>0$ such that $\left.J\right|_{\partial B_{\rho}} \geq a$. That is to say, $J$ satisfies the condition $\left(J_{1}\right)$ of the Mountain Pass Lemma.

For our setting, clearly $J(0)=0$. In order to exploit the Mountain Pass Lemma in critical point theory, we need to verify all other conditions of the Mountain Pass Lemma. By Lemma 2.2, $J$ satisfies the P.S. condition. So it suffices to verify the condition $\left(J_{2}\right)$.

From the proof of the P.S. condition in Lemma 2.2, we know

$$
J(u) \leq \frac{1}{2}\left(\bar{p} \lambda_{k}+\bar{q} \tilde{\lambda}_{k}\right)\|u\|^{2}-a_{1} c_{1}^{\beta}\|u\|^{\beta}+a_{2} k .
$$

Since $\beta>2$, we can choose $\bar{u}$ large enough to ensure that $J(\bar{u})<0$.

By the Mountain Pass Lemma, $J$ possesses a critical value $c \geq a>0$, where

$$
c=\inf _{h \in \Gamma} \sup _{s \in[0,1]} J(h(s))
$$

and

$$
\Gamma=\left\{h \in C\left([0,1], \mathbf{R}^{k}\right) \mid h(0)=0, h(1)=\bar{u}\right\} .
$$


Let $\tilde{u} \in \mathbf{R}^{k}$ be a critical point associated to the critical value $c$ of $J$, i.e., $J(\tilde{u})=c$. Similar to the proof of the P.S. condition, we know that there exists $\hat{u} \in \mathbf{R}^{k}$ such that

$$
J(\hat{u})=c_{\max }=\max _{s \in[0,1]} J(h(s)) .
$$

Clearly, $\hat{u} \neq 0$. If $\tilde{u} \neq \hat{u}$, then the conclusion of Theorem 1.2 holds. Otherwise, $\tilde{u}=\hat{u}$. Then $c=J(\tilde{u})=c_{\max }=\max _{s \in[0,1]} J(h(s))$. That is,

$$
\sup _{u \in \mathbf{R}^{k}} J(u)=\inf _{h \in \Gamma} \sup _{s \in[0,1]} J(h(s)) .
$$

Therefore,

$$
c_{\max }=\max _{s \in[0,1]} J(h(s)), \forall h \in \Gamma .
$$

By the continuity of $J(h(s))$ with respect to $s, J(0)=0$ and $J(\bar{u})<0$ imply that there exists $s_{0} \in(0,1)$ such that

$$
J\left(h\left(s_{0}\right)\right)=c_{\max } .
$$

Choose $h_{1}, h_{2} \in \Gamma$ such that $\left\{h_{1}(s) \mid s \in(0,1)\right\} \cap\left\{h_{2}(s) \mid s \in(0,1)\right\}$ is empty, then there exists $s_{1}, s_{2} \in(0,1)$ such that

$$
J\left(h_{1}\left(s_{1}\right)\right)=J\left(h_{2}\left(s_{2}\right)\right)=c_{\max } .
$$

Thus, we get two different critical points of $J$ on $\mathbf{R}^{k}$ denoted by

$$
u^{1}=h_{1}\left(s_{1}\right), u^{2}=h_{2}\left(s_{2}\right) .
$$

The above argument implies that the BVP (1.1) with (1.2) possesses at least two nontrivial solutions. The proof of Theorem 1.2 is finished.

\subsection{Proof of Theorem 1.3}

Proof. We only need to find at least one critical point of the functional $J$ defined as in (2.5).

By $\left(F_{5}^{\prime}\right)$, for any $u=\left(u_{1}, u_{2}, \cdots, u_{k}\right)^{*} \in \mathbf{R}^{k}$, we have

$$
\begin{aligned}
J(u) & =\frac{1}{2} \sum_{n=-1}^{k} p_{n+1}\left(\Delta^{2} u_{n}\right)^{2}+\frac{1}{2} \sum_{n=0}^{k} q_{n+1}\left(\Delta u_{n}\right)^{2}-\sum_{n=1}^{k} F\left(n, u_{n+1}, u_{n}\right) \\
& \geq \frac{1}{2} \underline{p} \sum_{n=-1}^{k}\left(u_{n+2}-2 u_{n+1}+u_{n}\right)^{2}+\frac{1}{2} \underline{q} \sum_{n=0}^{k}\left(u_{n+1}-u_{n}\right)^{2}-a_{3} \sum_{n=1}^{k}\left(\sqrt{u_{n+1}^{2}+u_{n}^{2}}\right)^{\alpha}-a_{4} k \\
& =\frac{1}{2} \underline{p} u^{*} P u+\frac{1}{2} \underline{q} u^{*} Q u-a_{3}\left\{\left[\sum_{n=1}^{k}\left(\sqrt{u_{n+1}^{2}+u_{n}^{2}}\right)^{\alpha}\right]^{\frac{1}{\alpha}}\right\}^{\alpha}-a_{4} k \\
& \geq \frac{1}{2} \underline{p} \lambda_{1}\|u\|^{2}+\frac{1}{2} \underline{q}_{1}\|u\|^{2}-a_{3} c_{2}^{\alpha}\left\{\left[\sum_{n=1}^{k}\left(u_{n+1}^{2}+u_{n}^{2}\right)\right]^{\frac{1}{2}}\right\}^{\alpha}-a_{4} k \\
\geq & \frac{1}{2}\left(\underline{p} \lambda_{1}+\underline{q} \tilde{\lambda}_{1}\right)\|u\|^{2}-2^{\alpha} a_{3} c_{2}^{\alpha}\|u\|^{\alpha}-a_{4} k \\
& \rightarrow+\infty \text { as }\|u\| \rightarrow+\infty .
\end{aligned}
$$

By the continuity of $J$, we know from the above inequality that there exist lower bounds of values of the functional. And this means that $J$ attains its minimal value at some 
point which is just the critical point of $J$ with the finite norm.

\subsection{Proof of Theorem 1.4}

Proof. Assume, for the sake of contradiction, that the BVP (1.1) with (1.2) has a nontrivial solution. Then $J$ has a nonzero critical point $u^{\star}$. Since

$$
\frac{\partial J}{\partial u_{n}}=\Delta^{2}\left(p_{n-1} \Delta^{2} u_{n-2}\right)-\Delta\left(q_{n} \Delta u_{n-1}\right)-f\left(n, u_{n+1}, u_{n}, u_{n-1}\right)
$$

we get

$$
\begin{aligned}
& \sum_{n=1}^{k} f\left(n, u_{n+1}^{\star}, u_{n}^{\star}, u_{n-1}^{\star}\right) u_{n}^{\star}=\sum_{n=1}^{k}\left[\Delta^{2}\left(p_{n-1} \Delta^{2} u_{n-2}^{\star}\right)-\Delta\left(q_{n} \Delta u_{n-1}^{\star}\right)\right] u_{n}^{\star} \\
& (3.1) \quad=\sum_{n=-1}^{k} p_{n+1}\left(\Delta^{2} u_{n}^{\star}\right)^{2}+\sum_{n=0}^{k} q_{n+1}\left(\Delta u_{n}^{\star}\right)^{2} \leq 0 .
\end{aligned}
$$

On the other hand, it follows from $\left(F_{6}\right)$ that

$$
\sum_{n=1}^{k} f\left(n, u_{n+1}^{\star}, u_{n}^{\star}, u_{n-1}^{\star}\right) u_{n}^{\star}>0 .
$$

This contradicts (3.1) and hence the proof is complete.

\section{Examples}

As an application of Theorems 1.2, 1.3 and 1.4, we give three examples to illustrate our main results.

Example 4.1. For $n \in \mathbf{Z}(1, k)$, assume that

$$
\Delta^{4} u_{n-2}-\Delta\left(9^{n} \Delta u_{n-1}\right)=\beta u_{n}\left[\varphi(n)\left(u_{n+1}^{2}+u_{n}^{2}\right)^{\frac{\beta}{2}-1}+\varphi(n-1)\left(u_{n}^{2}+u_{n-1}^{2}\right)^{\frac{\beta}{2}-1}\right],
$$

with boundary value conditions (1.2), where $\beta>2, \varphi$ is continuously differentiable and $\varphi(n)>0, n \in \mathbf{Z}(1, k)$ with $\varphi(0)=0$.

We have

$$
p_{n} \equiv 1, q_{n}=9^{n}, f\left(n, v_{1}, v_{2}, v_{3}\right)=\beta v_{2}\left[\varphi(n)\left(v_{1}^{2}+v_{2}^{2}\right)^{\frac{\beta}{2}-1}+\varphi(n-1)\left(v_{2}^{2}+v_{3}^{2}\right)^{\frac{\beta}{2}-1}\right]
$$

and

$$
F\left(n, v_{1}, v_{2}\right)=\varphi(n)\left(v_{1}^{2}+v_{2}^{2}\right)^{\frac{\beta}{2}}
$$

It is easy to verify all the assumptions of Theorem 1.2 are satisfied and then the BVP (4.1) with (1.2) possesses at least two nontrivial solutions.

Example 4.2. For $n \in \mathbf{Z}(1, k)$, assume that

$$
\Delta^{2}\left(8^{n-1} \Delta^{2} u_{n-2}\right)-\Delta\left(6^{n} \Delta u_{n-1}\right)=\alpha u_{n}\left[\psi(n)\left(u_{n+1}^{2}+u_{n}^{2}\right)^{\frac{\alpha}{2}-1}+\psi(n-1)\left(u_{n}^{2}+u_{n-1}^{2}\right)^{\frac{\alpha}{2}-1}\right],
$$

with boundary value conditions (1.2), where $1<\alpha<2, \psi$ is continuously differentiable and $\psi(n)>0, n \in \mathbf{Z}(1, k)$ with $\psi(0)=0$.

We have

$$
p_{n}=8^{n}, q_{n}=6^{n}, f\left(n, v_{1}, v_{2}, v_{3}\right)=\alpha v_{2}\left[\psi(n)\left(v_{1}^{2}+v_{2}^{2}\right)^{\frac{\alpha}{2}-1}+\psi(n-1)\left(v_{2}^{2}+v_{3}^{2}\right)^{\frac{\alpha}{2}-1}\right]
$$


and

$$
F\left(n, v_{1}, v_{2}\right)=\psi(n)\left(v_{1}^{2}+v_{2}^{2}\right)^{\frac{\alpha}{2}} .
$$

It is easy to verify all the assumptions of Theorem 1.3 are satisfied and then the BVP (4.2) with (1.2) possesses at least one solution.

Example 4.3. For $n \in \mathbf{Z}(1, k)$, assume that

$$
-\Delta^{4} u_{n-2}+\Delta\left(7^{n} \Delta u_{n-1}\right)=\frac{8}{5} u_{n}\left[\left(u_{n+1}^{2}+u_{n}^{2}\right)^{-\frac{1}{5}}+\left(u_{n}^{2}+u_{n-1}^{2}\right)^{-\frac{1}{5}}\right],
$$

with boundary value conditions (1.2).

We have

$$
p_{n} \equiv-1, q_{n}=-7^{n}, f\left(n, v_{1}, v_{2}, v_{3}\right)=\frac{8}{5} v_{2}\left[\left(v_{1}^{2}+v_{2}^{2}\right)^{-\frac{1}{5}}+\left(v_{2}^{2}+v_{3}^{2}\right)^{-\frac{1}{5}}\right]
$$

and

$$
F\left(n, v_{1}, v_{2}\right)=\left(v_{1}^{2}+v_{2}^{2}\right)^{\frac{4}{5}} .
$$

It is easy to verify all the assumptions of Theorem 1.4 are satisfied and then the BVP (4.3) with (1.2) has no nontrivial solutions.

\section{References}

[1] C.D. Ahlbrandt. Dominant and recessive solutions of symmetric three term recurrences. J. Differential Equations, 107(2) (1994) 238-258.

[2] V. Anuradha, C. Maya and R. Shivaji. Positive solutions for a class of nonlinear boundary value problems with Neumann-Robin boundary conditions. J. Math. Anal. Appl., 236(1) (1999) 94-124.

[3] D. Arcoya. Positive solutions for semilinear Dirichlet problems in an annulus. J. Differential Equations, 94(2) (1991) 217-227.

[4] X.C. Cai, J.S.Yu and Z.M. Guo. Existence of periodic solutions for fourth-order difference equations. Comput. Math. Appl., 50(1-2) (2005) 49-55.

[5] M. Cecchi, M. Marini and G. Villari. On the monotonicity property for a certain class of second order differential equations. J. Differential Equations, 82(1) (1989) 15-27.

[6] S.Z. Chen. Disconjugacy, disfocality, and oscillation of second order difference equations. J. Differential Equations, 107(2) (1994) 383-394.

[7] P. Chen and H. Fang. Existence of periodic and subharmonic solutions for second-order p-Laplacian difference equations. Adv. Difference Equ., 2007 (2007) 1-9.

[8] P. Chen and X.H. Tang. Existence of infinitely many homoclinic orbits for fourth-order difference systems containing both advance and retardation. Appl. Math. Comput., 217(9) (2011) 4408-4415.

[9] P. Chen and X.H. Tang. New existence and multiplicity of solutions for some Dirichlet problems with impulsive effects. Math. Comput. Modelling, 55(3-4) (2012) 723-739.

[10] H. Fang and D.P. Zhao. Existence of nontrivial homoclinic orbits for fourthorder difference equations. Appl. Math. Comput., 214(1) (2009) 163-170.

[11] C.J. Guo, D. O'Regan, Y.T. Xu and R.P. Agarwal. Existence and multiplicity of homoclinic orbits of a second-order differential difference equation via variational methods. Appl. Math. Inform. Mech., 4(1) (2012) 1-15.

[12] Z.M. Guo and J.S. Yu. Applications of critical point theory to difference equations. Fields Inst. Commun., 42 (2004) 187-200. 
[13] Z.M. Guo and J.S. Yu. Existence of periodic and subharmonic solutions for second-order superlinear difference equations. Sci. China Math, 46(4) (2003) 506-515.

[14] Z.M. Guo and J.S. Yu. The existence of periodic and subharmonic solutions of subquadratic second order difference equations. J. London Math. Soc., 68(2) (2003) 419-430.

[15] J.K. Hale and J. Mawhin. Coincidence degree and periodic solutions of neutral equations. J. Differential Equations, 15(2) (1974) 295-307.

[16] J. Henderson and H.B. Thompson. Existence of multiple solutions for secondorder discrete boundary value problems. Comput. Math. Appl., 43(10-11) (2002) 1239-1248.

[17] V.L. Kocic and G. Ladas. Global Behavior of Nonlinear Difference Equations of Higher Order with Applications. Kluwer Academic Publishers, Dordrecht (1993).

[18] Y.J. Liu and W.G. Ge. Twin positive solutions of boundary value problems for finite difference equations with p-Laplacian operator. J. Math. Anal. Appl., 278(2) (2003) 551-561.

[19] H. Matsunaga, T. Hara and S. Sakata. Global attractivity for a nonlinear difference equation with variable delay. Computers Math. Appl., 41(5-6) (2001) 543-551.

[20] J. Mawhin and M. Willem. Critical Point Theory and Hamiltonian Systems. Springer, New York (1989).

[21] R.E. Mickens. Difference Equations: Theory and Application. Van Nostrand Reinhold, New York (1990).

[22] A. Pankov and N. Zakhrchenko. On some discrete variational problems. Acta Appl. Math., 65(1-3) (2001) 295-303.

[23] A. Peterson and J. Ridenhour. The (2,2)-disconjugacy of a fourth order difference equation. J. Difference Equ. Appl., 1(1) (1995) 87-93.

[24] J. Popenda and E. Schmeidel. On the solutions of fourth order difference equations. Rocky Mountain J. Math., 25(4) (1995) 1485-1499.

[25] P.H. Rabinowitz. Minimax Methods in Critical Point Theory with Applications to Differential Equations. Amer. Math. Soc., Providence, RI, New York (1986).

[26] Y. Rodrigues. On nonlinear discrete boundary value problems. J. Math. Anal. Appl., 114(2) (1986) 398-408.

[27] A.N. Sharkovsky, Y.L. Maistrenko and E.Y. Romanenko. Difference Equations and Their Applications. Kluwer Academic Publishers, Dordrecht (1993).

[28] H.P. Shi, W.P. Ling, Y.H. Long and H.Q. Zhang. Periodic and subharmonic solutions for second order nonlinear functional difference equations. Commun. Math. Anal., 5(2) (2008) 50-59.

[29] E. Thandapani and I.M. Arockiasamy. Fourth-order nonlinear oscillations of difference equations. Comput. Math. Appl., 42(3-5) (2001) 357-368.

[30] H.Y. Wang. On the existence of positive solutions for semilinear elliptic equations in the annulus. J. Differential Equations, 109(1) (1994) 1-7.

[31] J. Yan and B. Liu. Oscillatory and asymptotic behavior of fourth order nonlinear difference equations. Acta. Math. Sinica, 13(1) (1997) 105-115.

[32] J.S. Yu and Z.M. Guo. Boundary value problems of discrete generalized EmdenFowler equation. Sci. China Math, 49(10) (2006) 1303-1314.

[33] J.S. Yu and Z.M. Guo. On boundary value problems for a discrete generalized Emden-Fowler equation. J. Differential Equations, 231(1) (2006) 18-31.

[34] Z. Zhou, J.S. Yu and Y.M. Chen. Periodic solutions of a 2nth-order nonlinear difference equation. Sci. China Math, 53(1) (2010) 41-50. 
[35] W.M. Zou and M. Schechter. Critical Point Theory and Its Applications. Springer, New York (2006). 